Notes

\title{
Determination of Pantothenic Acid in an Elemental Diet by Column-Switching High-Performance Liquid Chromatography with Ultraviolet Detection
}

\author{
Hiroshi IWASE \\ Ajinomoto Co., Inc., Kawasaki Factory, Suzuki-cho, Kawasaki 210, Japan
}

Keywords High-performance liquid chromatography, column-switching, ultraviolet detection, pantothenic acid, elemental diet

Numerous techniques, including pre- and postcolumn labeling and column-switching, have been developed in order to extend the application of high-performance liquid chromatography (HPLC). Column-switching is a simple and rapid technique which has been applied to complex sample matrices, such as the analysis of drugs as well as their metabolites in biological fluids. ${ }^{1,2}$

The routine analysis of pantothenic acid in an elemental diet (commercial name Elental, Ajinomoto, Kawasaki), which comprises a mixture of 46 compounds (e.g. amino acids, vitamins, organic acids, soybean oil, dextrin, minerals $)^{3}$, has proven to be valuable for process control, quality control and clinical chemistry.

In almost all cases, microbiological methods ${ }^{4}$ have generally been used for the routine determination of vitamins. However, these methods are both tedious and time-consuming. ${ }^{3,4}$ Calcium pantothenate was analyzed by titration ${ }^{6,7}$ with ethylenediaminetetraacetic acid disodium salt. Titration is not suitable for such complex sample matrices as Elental. HPLC has been used for the analysis of vitamins. The analysis of pantothenic acid in an elemental diet has not been investigated in detail.

In previous studies ${ }^{5,8-10}$ cyanocobalamin, ascorbic acid, folic acid, nicotinamide and pyridoxine in Elental were determined by HPLC with visible detection at $550 \mathrm{~nm}$, electrochemical detection at $70 \mathrm{mV}$ (versus an $\mathrm{Ag} / \mathrm{AgCl}$ reference electrode) and UV detection at 260 or $290 \mathrm{~nm}$.

Since pantothenic acid has no specific spectra in the UV region, a selective analysis of pantothenic acid was not possible. The maximum concentration of the amino acids was 1620 -times higher than that of pantothenic acid. It was proven that the removal of coexising interference caused by complex sample matrices was valuable for the analysis of pantothenic acid in Elental.

A previous paper ${ }^{10}$ reported the determination of nicotinamide and pyridoxine in Elental by columnswitching HPLC with detection at 260 or $290 \mathrm{~nm}$, since the column-switching technique is both simple and rapid.
The present paper concerns a simple routine determination of pantothenic acid (as calcium salt, $14.9 \mu \mathrm{g} / \mathrm{g}$ ) in Elental. A routine determination was performed by column-switching HPLC with UV detection at $210 \mathrm{~nm}$. This paper also describes the determination of the abovementioned vitamin in an additional two elemental diets: Elental $\mathbf{P}$ for pediatrics and Hepan ED for hepatic failure.

\section{Experimental}

\section{Materials}

Elental, Elental P and Hepan ED were obtained from Ajinomoto Co. (Kawasaki). The calcium pantothenate used in this study was of Japanese Pharmacopeia standard. Sodium 1-heptanesulfonate was obtained from Aldrich Co. (WI, USA). Acetonitrile (Wako Pure Chemical, Osaka) was of HPLC grade. The other reagents were all of analytical grade.

\section{Sample preparations}

To a solution of Elental $(20 \mathrm{~g})$ dissolved completely in deionized water $(60 \mathrm{ml})$ on a water bath at $50^{\circ} \mathrm{C}$ was added sodium chloride $(10 \mathrm{~g})$. After this solution was allowed to stand at room temperature for $30 \mathrm{~min}$, it was accurately diluted to $100 \mathrm{ml}$ with deionized water: this solution was then extracted with hexan $(10 \mathrm{ml})$ for $3 \mathrm{~min}$ to remove any oils. The aqueous layer was used for a test sample. An aliquot $(20 \mu \mathrm{l})$ was injected into the chromatograph.

Elental $\mathbf{P}$ and Hepan ED were also prepared in the same manner as described above concerning Elental.

Pantothenic acid was stable in aqueous solution at $50^{\circ} \mathrm{C}$ for $1 \mathrm{~h}$.

\section{High-performance liquid chromatography}

Two Model 655A-11 high-performance liquid chromatographs (Hitachi Ltd., Tokyo) equipped with two Model 655A variable-wavelength detectors (Hitachi Ltd., Tokyo) set at $210 \mathrm{~nm}$ and a Model HPV 6A column 
switching device (Gaskuro Kogyo, Co., Tokyo) were used. Capcellpak $\mathrm{C}_{18}(5 \mu \mathrm{m})$ (Shiseido Co. Tokyo) $(15 \times 0.46 \mathrm{~cm}$ i.d.) and $(25 \times 0.46 \mathrm{~cm}$ i.d. $)$ were used as the precolumn and analytical column, respectively. The samples were applied by a Rheodyne Model 7125 sample loop injector with an effecive volume of $20 \mu \mathrm{l}$. A Shimadzu UV variable recording spectrophotometer (UV-2100; Shimadzu Co., Kyoto) was used for the absorption spectra.

\section{Chromatographic conditions}

After injection of a $20 \mu \mathrm{l}$ sample solution into the precolumn, which had previously been equilibrated with acetonitrile-water ( $\mathrm{pH} 2.1$, adjusted with phosphoric acid) $(5: 95)$, the column was washed for 4 min with the above-mentioned mobile phase at a flow rate of $1.2 \mathrm{ml} /$ min. The substances adsorbed on the precolumn were introduced to the analytical column with acetonitrilewater $(\mathrm{pH} 2.1$, adjusted with phosphoric acid) $(9: 91)$ with $1.5 \mathrm{mM}$ sodium 1-heptansulfonate for $0.75 \mathrm{~min}$ at a flow rate of $0.6 \mathrm{ml} / \mathrm{min}$ and a column temperature of $35^{\circ} \mathrm{C}$ by switching a six-port valve. After introducing the substances to the analytical column, the six-port valve was returned to its original position. The precolumn was then washed with acetonitrile-water $(\mathrm{pH} 2.1)(5: 95)$ in preparation for the next injection.

\section{Results and Discussion}

\section{Chromatography}

First effort was focused on an analysis of pantothenic acid in Elental using only one analytical column, without the use of column-switching method with UV detection at $210 \mathrm{~nm}$. However, pantothenic acid could not be identified, since many co-existing compounds (which might be considered to be amino acids with a maximum concentration 1.620-times higher than that of pantothenic acid) were observed on the chromatogram.

Next effort was focused on the clean-up of Elental using any ion-exchange resin column (AG-50W-X4, Bio-Rad Co., CA, USA, $7 \mathrm{ml}, \mathrm{H}^{+}$form) so as to retain any interference, such as amino acids, using only one analytical column without the use of the columnswitching method. The combined solution of effluent and washings was concentrated followed by HPLC with UV detection at $210 \mathrm{~nm}$. By using this clean-up method, the peak of pantothenic acid could be identified with sufficient intensity. However, this technique was not suitable for a simple routine analysis of pantothenic acid in Elental. It was both tedious and time-consuming, since it requiers a long elution time of pantothenic acid as well as an additional and concentration of the effluent and washings.

Further effort was focused on the analysis of pantothenic acid in Elental by column-switching HPLC with detection at $210 \mathrm{~nm}$.

At the beginning of the work, the effect of the length of precolumns $(3 \times 0.46 \mathrm{~cm}$ i.d., $15 \times 0.46 \mathrm{~cm}$ i.d. $)$ on the

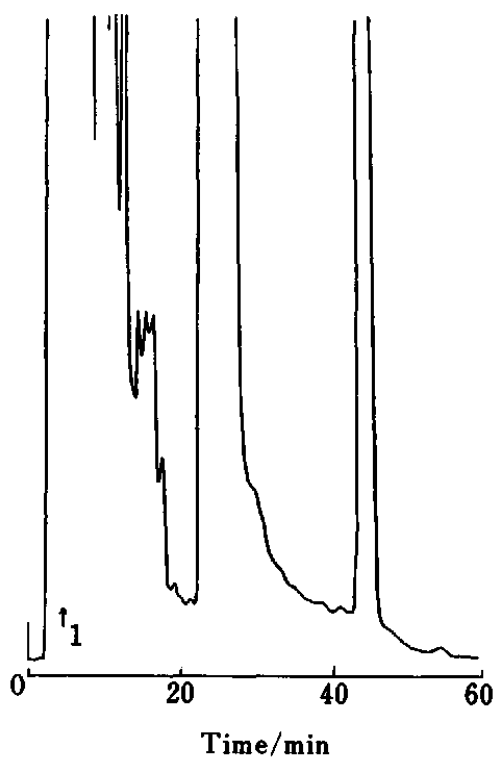

Fig. 1 Elution of pantothenic acid in Elental on a precolumn $(15 \times 0.46 \mathrm{~cm}$ i.d.) with detection at $210 \mathrm{~nm}$. Mobile phase: acetonitrile-water ( $\mathrm{pH} 2.1$, adjusted with phosphoric acid) $(5: 95)$ at a flow rate of $1.2 \mathrm{ml} / \mathrm{min}$. 1: pantothenic acid.

separation of pantothenic acid was examined.

The first choice was a $3 \times 0.46 \mathrm{~cm}$ i.d. precolumn. Using this column, pantothenic acid could not be completely separated on the analytical column. Second choice was a $15 \times 0.46 \mathrm{~cm}$ i.d. precolumn. Using this column, pantothenic acid could be separated on the analytical column. Thus, the use of a $15 \times 0.46 \mathrm{~cm}$ i.d. precolumn was undertaken in this study.

The retention times of standard pantothenic acid, and the column connection times from precolumn to analytical column were examined for the analysis of this vitamin as well as the elimination of any interference caused by complex sample matrices.

A chromatogram of Elental by HPLC with UV detection at $210 \mathrm{~nm}$ on a $15 \times 0.46 \mathrm{~cm}$ i.d. precolumn is shown in Fig. 1. The peak of pantothenic acid was observed at a retention time of $c a .4 \mathrm{~min}$. Complete elution requires about $60 \mathrm{~min}$ (Fig. 1).

After washing for $4 \mathrm{~min}$ with acetonitrile-water (pH 2.1) (5:95), the precolumn and analytical column were connected for various periods in order to examine the connection time.

When the connection times were a made shorter than for $0.75 \mathrm{~min}$, the peak of pantothenic acid was smaller; when the connection times was a made longer than for 0.75 min, many other unknown overloading peaks were observed, pantothenic acid could not be identified. The column connection time of this vitamin was therefore set at $0.75 \mathrm{~min}$.

The chromatogram of pantothenic acid in Elental shown in Fig. 2 (detection limit: $c a .5 \mathrm{ng}(S / N=2)$ ) was obtained by column-switching HPLC with detection at $210 \mathrm{~nm}$. Pantothenic acid was separated completely, 


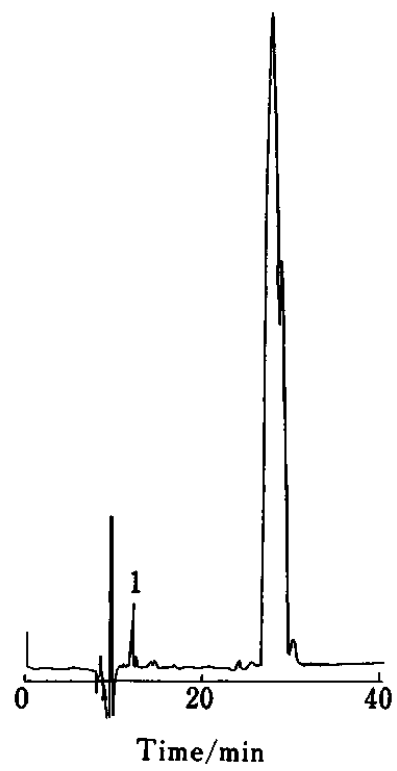

Fig. 2 Chromatogram of pantothenic acid in Elental by column-switching HPLC with UV detection at $210 \mathrm{~nm}$ Amount of pantothenic acid injected: $57.2 \mathrm{ng}$ in $20 \mu \mathrm{l}$. 1: pantothenic acid.

irrespective of the sample containing many coexisting compounds and monitoring of a nonspecific spectrum at $210 \mathrm{~nm}$.

In order to identify pantothenic acid in Elental, a freshly prepared model solution containing 45 compounds with no addition of pantothenic acid was examined by this method. Since no peak of pantothenic acid was observed on the chromatogram, the procedure mentioned here might be considered to be advantageous for any routine determination of pantothenic acid in a complex mixture.

\section{Determination of pantothenic acid}

A calibration graph for pantothenic acid (as calcium salt) was constructed by plotting the peak height against the amount of pantothenic acid; satisfactory linearity was obtained within the range 0 to $0.12 \mu \mathrm{g}$.

A known amount of pantothenic acid was added to Elental and the overall recovery was estimated by the standard addition method. Pantothenic acid was recovered at about $95 \%$. The relative standard deviations (RSD) of within-day $(n=5)$ and between-day assays $(n=5)$ with no addition of pantothenic acid were 1.8 and $2.7 \%$, respectively.

The results given in Table 1 list the analytical data for
Table 1 Analytical data for calcium pantothenate in three elemental diets

\begin{tabular}{lccc}
\hline Elemental diet & $\begin{array}{c}\text { Concentration } \\
\text { indicated } \\
(\mathrm{mg} / 100 \mathrm{~g})\end{array}$ & $\begin{array}{c}\text { Analytical } \\
\text { data } \\
(\mathrm{mg} / 100 \mathrm{~g})\end{array}$ & Recovery, \% \\
\hline Elental $^{\mathrm{a}}$ & 1.49 & 1.43 & 98.0 \\
& & 1.42 & 95.3 \\
Elental P & 2.48 & 1.42 & 95.3 \\
Hepan ED & 2.06 & 2.36 & 95.2 \\
\hline
\end{tabular}

a. RSD of within-day: $1.8 \%(n=5)$ with no addition of calcium pantothenate.

pantothenic acid in Elental, Elental $\mathbf{P}$ and Hepan ED. There is good agreement between the indicated and found concentrations of pantothenic acid.

In conclusion, this method is satisfactory with respect to both simplicity and reproducibility. Since it is simple and convenient, it is therefore applicable to any routine analysis of pantothenic acid in Elental, Elental $\mathbf{P}$ or Hepan ED. The application of the proposed method to the determination of drugs in biological fluids is also being studied.

\section{References}

1. K. Matsumoto, H. Kikuchi and H. Iri, J. Chromatogr., 425, 323 (1989).

2. A. Mikan, J. M. Lanan, F. G. Lopez and A. D-G. Hurle, Biomed. Chromatogr., 4, 154 (1990).

3. S. Ogoshi (ed.), "Elental Diet", Nankodo Publishing, Tokyo, 1983.

4. T. Suzuki and S. Muraoka, "Bitamine Aminosan no Biseibutsu Teiryoho", p. 101, Nanzando Publishing, Tokyo, 1956.

5. H. Iwase, J. Chromatogr., 590, 187 (1992).

6. "The United States Pharmacopeia", XXII Revision, pp. 944, 1194, US Pharmacopeia Convention, Rockville, 1990.

7. "The Pharmacopeia of Japan", 12 th ed., p. C-1732, Hirokawa Publishing, Tokyo, 1992.

8. H. Iwase, J. Chromatogr., 606, 277 (1992).

9. H. Iwase, J. Chromatogr., 609, 399 (1992).

10. H. Iwase, J. Chromatogr., 625, 377 (1992).

(Received September 1, 1992) (Accepted November 26, 1992) 